\title{
The large effect of a small bias field in liquid crystal magnetic transitions
}

\author{
Nina Podoliak ${ }^{1}$, Oleksandr Buchnev ${ }^{1}$, Giampaolo D’Alessandro ${ }^{2}$, Malgosia Kaczmarek ${ }^{1}$ and Timothy J. Sluckin $^{2}$ \\ ${ }^{1}$ School of Physics and ${ }^{2}$ School of Mathematics, University of Southampton, Southampton SO17 1BJ, England, UK
}

(Dated: July 30, 2010)

\begin{abstract}
Most liquid crystals show low sensitivity to magnetic field. However, in this letter we show that a small bias magnetic field not only breaks the symmetry of the ground state, but also plays a crucial role in facilitating the reorientation induced by a large test magnetic field. In particular, a small bias field may alter significantly the strength of the test field needed to observe a given reorientation of the liquid crystal. Moreover, the bias field interacts with other symmetry breaking features of the cell, e.g. pretilt, to change also the qualitative features of the equilibrium state.
\end{abstract}

PACS numbers: 61.30.Gd, 64.70.M-, 42.70.Df,42.79.Kr

The Frederiks effect underlies most modern liquid crystal devices [1]. In the original Frederiks experiments [2] a liquid crystal is placed in a cell subject to a homogeneous planar surface alignment. A magnetic field, applied perpendicular to the cell plane, couples to the (liquid crystal) director. But, as a result of liquid crystal elasticity and surface anchoring effects, the field only begins to reorient the liquid crystal director above a critical Frederiks threshold. At the threshold the symmetry of the system is spontaneously broken, and the director may tilt toward the favored direction in one of two ways.

Modern devices use electric rather than magnetic fields, because the dielectric coupling is much stronger than the magnetic coupling. Recently, however, given the prospect of ferronematic devices based on magnetic nanoparticle liquid crystal colloids [3-7], there has been a renaissance of interest in magnetic effects. In this letter we return to the original Frederiks picture.

From a device point of view, the Frederiks threshold symmetry-breaking is undesirable. Spatially inhomogeneous spontaneous symmetry-breaking causes defects at domain boundaries, which dominate the optical effects and ruin the devices. The traditional technique for avoiding this is to introduce a small surface pretilt $[1,8,9]$. This biases the switching in one sense rather than the other, formally destroying critical point associated with the symmetry-breaking. Ferronematic devices require another symmetry-breaking field, a so-called bias field [4], in the plane of the cell. This orders the ferromagnetic colloidal particles, enabling them to couple coherently with the liquid crystal director. The bias field also breaks the parity symmetry of the cell.

In this letter we study pure liquid crystal systems, subject to pretilt and bias fields which are much lower than the threshold field. These studies are an essential preliminary to the study of ferronematic systems, but yield interesting effects themselves. We find, both experimentally and theoretically, two striking results. First, at conventional surface tilts $\left(\simeq 2-4^{\circ}\right)$, small bias fields cause shifts in an effective Frederiks threshold which are numerically significantly larger than the magnitude of the bias field itself. Second, for any given pretilt there is a

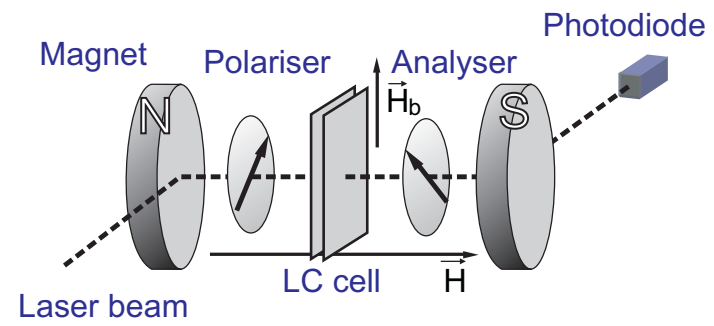

FIG. 1. Schematic of the experimental set up (see text).

value of the bias field which to linear order cancels out the pretilt. For this special bias field, the cell behaves almost like an ideal cell and new equilibrium states become accessible.

We start by describing the experimental set up and measurements of the effects of the bias field on the reorientation of the liquid crystals. We fit a simple, but realistic model to the experimental data and use it to quantify the bias field effect. We then use the model to extend the experimental results to large bias fields, where a Frederiks-like transition appears. We develop an approximate model to obtain some analytical expressions for the threshold and conclude the paper with an analysis of the results.

The magneto-optic response of the liquid crystal cell is measured using the following set up. The cell consists of a $50 \mu \mathrm{m}$ liquid crystal layer placed between two glass substrates covered with a polyimide layer, rubbed in opposite directions. The thickness is controlled by a spacer, and known to an accuracy of $0.5 \mu \mathrm{m}$, and further fixed more precisely by fitting optical transmission data. The surface treatment produces planar alignment of the liquid crystal molecules in the cell with a pretilt angle $\alpha=2.70^{\circ}$, as measured by the crystal rotation method [10]. The cell is placed between two crossed polarizers that form a $\pi / 4$ angle with the alignment direction (Figure 1). The main "test" magnetic field $\boldsymbol{H}$ (of strength up to $2000 \mathrm{G}$ ) is applied perpendicular to the cell plane. There is also a small bias field $\boldsymbol{H}_{\boldsymbol{b}}$, between 10 to 30 Gauss, along the initial alignment direction of the liquid crystal. The reorientation of the nematic molecules 
is studied by monitoring the intensity of a laser beam that passes through this system. In particular, we extract from this the phase lag between the ordinary and extraordinary components of the beam as a function of the test field intensity for various amplitudes of the bias field. It is this quantity that is fitted by the model and used to study the effect of the bias field on the much larger test field.

This system is modeled using the standard FrankOseen theory [1] assuming strong anchoring. We indicate with $0<z<1$ the dimensionless coordinate into the cell thickness (scaled with the thickness $D$ of the cell) and we assume that the cell is infinitely extended in the other two directions, so that the director field is a function of the $z$-coordinate only. Moreover, we also assume that the director field lies in the plane of the cell and can be described by a unit vector $\boldsymbol{n}$ that forms an angle $\theta(z)$ with the cell facets. Under these approximations the alignment of the liquid crystal is the minimum of the non-dimensional free energy

$$
\begin{aligned}
F=\int_{0}^{1} & \frac{1}{2}\left(\frac{d \theta}{d z}\right)^{2}\left[1+p \sin ^{2} \theta\right]- \\
& -\frac{\pi^{2}}{2}[h \sin \theta+b \cos \theta]^{2} d z
\end{aligned}
$$

where $p=\left(K_{3}-K_{1}\right) / K_{1}$, with $\left\{K_{1}, K_{3}\right\}$ respectively the splay and bend elastic constants of the liquid crystal. The test, $H$, and bias, $H_{b}$, magnetic fields are represented by the non-dimensional fields $h=H / H_{s}$ and $b=H_{b} / H_{s}$ respectively, where $H_{s}=\pi \sqrt{K_{1} / \chi_{a}} D^{-1}$ and $\chi_{a}$ is the anisotropy of the diamagnetic permeability of the liquid crystal. In this units, the Frederiks transition with no bias field and no pretilt, $b=\alpha=0$, corresponds to $h=1$. In writing (1) we have adopted the convention that the bias field is positive if it points in the same direction as the director field $\boldsymbol{n}$ at the boundary of the cell. For the E7 liquid crystal used in the experiments $p \simeq 0.56$ and $H_{s} \simeq 1,920 \mathrm{G}$.

The Euler-Lagrange equation that minimizes the free energy (1), is solved using a spectral method [11] coupled with a Broyden non-linear solver [12]. From the resulting director field $\theta(z)$ we compute the phase lag $\Delta \varphi$ between the ordinary and extraordinary polarization using

$$
\begin{aligned}
& \Delta \varphi=k_{0} \int_{0}^{1}\left(n_{o}-n_{e f f}\right) d z, \\
& n_{e f f}=n_{e}\left[1+\nu \sin ^{2} \theta\right]^{-1 / 2},
\end{aligned}
$$

where $k_{0}=(2 \pi D) / \lambda$. Here $\lambda$ is the light wave length in vacuum, $n_{e, o}$ are the extraordinary and ordinary refractive index respectively and $\nu=\left(n_{e}^{2}-n_{o}^{2}\right) / n_{e}^{2}$. The experimental phase lag $\Delta \varphi_{e}$ is recovered from the experimental measurements of the normalized cross-polarized intensity $I_{\perp}$ using the relation

$$
I_{\perp}=\sin ^{2}\left(\frac{\Delta \varphi_{e}}{2}\right) .
$$

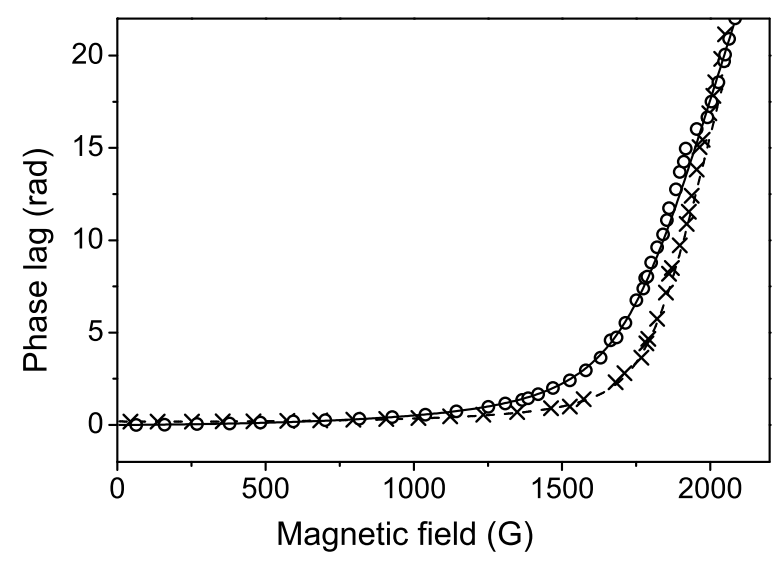

FIG. 2. Fit of the experimental $\Delta \varphi_{e}$ (symbols) and numerical $\Delta \varphi$ (lines) phase lag for bias field $H_{b}=30 \mathrm{G}$ (circles and solid line) and $H_{b}=-30 \mathrm{G}$ (crosses and dashed line).

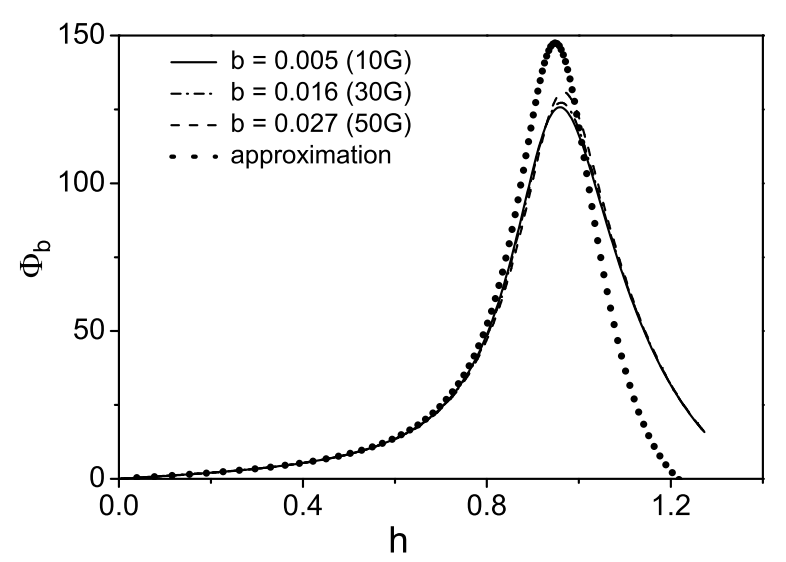

FIG. 3. The thin overlapping lines are a plot of the numerical estimates of $\Phi_{b}$, equation (4), for different values of the bias field. The thick dotted line is the plot of the fourth order approximation of $\Phi_{b}$, see equation (8) and text that refers to it.

The fit in Figure 2 shows clearly that the model (1) is well capable of fitting the experimental data. The only fitting parameters are the thickness of the cell (fixed by $\Delta \varphi_{e}$ at $h=0$ ) and the diamagnetic anisotropy $\chi_{a}$. All other parameters are either taken from the literature $\left(K_{1,3}\right.$ and $\left.n_{e, o}\right)$ or measured directly (pretilt angle, magnitude of the bias field).

In Figure 2 we see two phase-lag vs. test field curves with opposite bias fields. However, they resemble curves with shifted test-field scales. Surprisingly, the shift in the apparent test-field scale is significantly larger than the magnitude of the perpendicular bias field causing it. For example, the difference in test fields $H$ that correspond to $\Delta \varphi=3$ for $H_{b}= \pm 30 \mathrm{G}$ is approximately $200 \mathrm{G}$, an "amplification" of a factor of about 7. Indeed, 


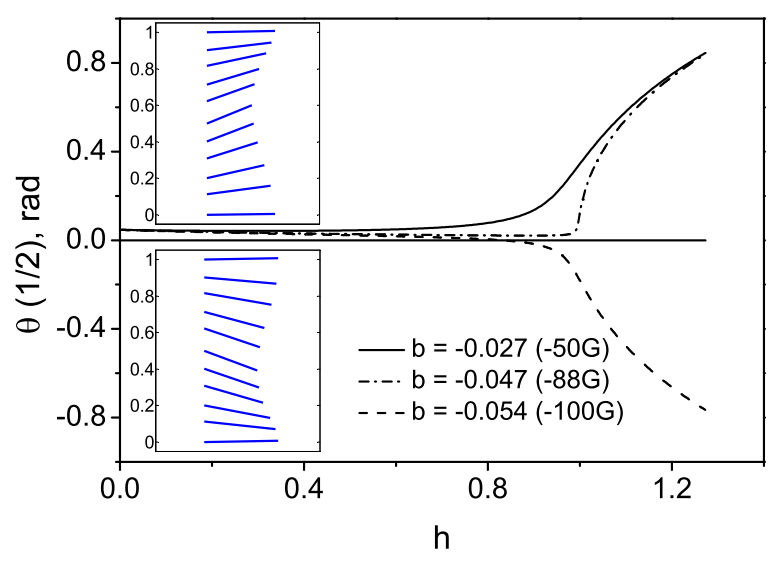

FIG. 4. Plot of the director field angle at the center of the cell as a function of the test field amplitude for different values of the bias field. The insets illustrate the director profile inside the cell for $h=1.27$ : the top one, for $b=-0.027$ $\left(H_{b}=-50 G\right)$, represents the synclinic configuration; the bottom one, for $b=-0.054\left(H_{b}=-100 G\right)$, represents the anticlinic configuration.

the language "bias field" that we use to describe the phenomenon is borrowed from the theory of transistors, and the analogy should be clear.

We characterize this effect using the function

$$
\Phi_{b}=\left.\frac{\Delta \varphi(h, b)-\Delta \varphi(h,-b)}{2 b} \simeq \frac{\partial \Delta \varphi}{\partial b}\right|_{b=0},
$$

i.e. the rate of change of the phase lag as a function of the bias field for constant test field. This quantity is plotted in Figure 3 for various values of the bias field. From this plot we see that the values of this function are universal up to fairly large values of the bias field. This universal behavior breaks down at sufficiently large negative values of the bias field.

To understand these changes in the behavior of the system we consider the plot of director field angle at the center of the cell shown in Figure 4. The inset to Figure 4 depicts the associated configurations, which can be either "synclinic" $(\theta(1 / 2)>\alpha$, with director tilt inclined toward the surface pretilt), or "anticlinic" $(\theta(1 / 2)<\alpha$, with director tilt opposing the surface pretilt). As negative bias field magnitude increases, the field-induced reorientation transition sharpens, in a manner resembling the classical Frederiks threshold. At this critical bias field, the director field reorientation mode (for high fields) switches from a synclinic to anticlinic configuration.

To determine the behavior of $\Phi_{b}$ and gain a better understanding of this new threshold behavior, we have looked for approximate solutions of the director field alignment. We substitute $\theta=\alpha+\hat{\theta}$, with $\alpha \ll 1$, in equation (1) with $p=0$. A first order expansion of the free energy in powers of $\alpha$ and $b$ yields

$$
\begin{aligned}
F= & \int_{0}^{1} \frac{1}{2}\left(\frac{d \hat{\theta}}{d z}\right)^{2}-\frac{\pi^{2}}{2} h^{2} \sin ^{2} \hat{\theta}- \\
& -\frac{\pi^{2}}{2} h(\alpha h+b) \sin 2 \hat{\theta} d z+O\left(\alpha^{2}, b^{2}\right) .
\end{aligned}
$$

To quantify this more precisely we assume that [13]

$$
\theta=\alpha+\psi \sin (\pi z)
$$

The free energy can then be minimized in terms of the amplitude $\psi$ of the modulation, subject to the condition that $\alpha, b, \psi \ll 1$. The quantity $\psi$ can be thought of as an order parameter for the Frederiks distortion. In leading order in $\alpha, b$, this yields

$$
\begin{aligned}
F \sim & -2 \pi(\alpha h+b) h \psi+\frac{\pi^{2}}{4}\left(1-h^{2}\right) \psi^{2}+ \\
& +\frac{\pi^{2}}{16} h^{2} \psi^{4}+O\left(\alpha^{2}, b^{2}, \psi^{5}\right) .
\end{aligned}
$$

Eq.(7) shows that the pretilt and the bias field have opposite effects. The geometry of this cell resembles that of the pi-cell $[14,15]$. Suppose the presence of surface pretilt and the test magnetic field. The director can in principle reorient either in the synclinic or anticlinic configuration (Figure 4). In the absence of a bias field, the synclinic mode always has lower energy.

An analysis of higher order terms in the free energy, not included in equation (7), shows that when a bias field is introduced at zero test field, the leading order coefficient of the $\psi$ term is now $-2 \pi \alpha b^{2}$. This always causes a slight anticlinic perturbation, with $\psi \sim-b^{2} \alpha$, independent of the direction of the bias field. Once the test field $h$ is introduced, however, the extra response in $\psi$ is now linear in $b$ and in $h$. A positive bias field favors the synclinic configuration, whereas a negative bias field relatively favors the anticlinic configuration.

The calculations are insensitive to the value of $p$ for low distortions, as the free energy in eq.(1) depends on $p \sin ^{2} \theta$, and we find this in our calculations. In the order parameter expansion eq.(7), the leading order terms containing $p$ are $O\left(p \alpha^{2} \psi^{2}\right)$ and $O\left(p \alpha \psi^{3}\right)$, showing explicitly that close to the Frederiks threshold, the properties of the system depends only weakly on $p$.

We have also made explicit calculations for strong but finite anchoring. So long as the ratio $\epsilon=\pi K / W D \ll 1$, we find only a weak extra effect. For identical walls, in the spirit of eq.(7), it is possible to introduce a new surface distortion $\vartheta_{s}$, which measures the departure of the surface angle from its ideal value. This is then coupled in the free energy expansion to $\psi$. We can show analytically that in this case, in scaled units, the ideal Frederiks threshold behaves as $1-2 \epsilon / \pi$.

Spontaneous Frederiks reorientation is triggered when the coefficient of the quadratic term in eq.(7) changes sign 
at $h \approx 1$. This reorientation will be synclinic/anticlinic depending on the sign of the linear term in $\psi$ at $h=1$. The transition between synclinic and anticlinic relaxation thus takes place at $b=-\alpha$.

From a symmetry and dynamical systems point of view the Frederiks transition for $\alpha=b=0$ is a pitchfork bifurcation [16]. The zero tilt solution becomes unstable with increasing test field and two solutions, with opposite non-zero tilt, appear. The non-zero pretilt angle acts as a perturbation. This bifurcation unfolds into two branches. The stable synclinic branch is smoothly connected to the zero field solution. The unstable saddle-node branch is no longer smoothly accessible under experimental conditions and corresponds to the anticlinic solution. The bias field unfolds this bifurcation further. The anticlinic branch now becomes smoothly connected to the zero field solution and, hence, experimentally accessible, while the synclinic branch is now separated from the zero field solution by a potential barrier. The reconnection occurs at $b=-\alpha$, at which point the bias field has in some sense just "canceled out" the effect of the pretilt.

It is interesting to note that, even when the bias field forces the Frederiks relaxation to follow the anticlinic route, for sufficiently high test fields the synclinic configuration possesses lower energy than the anticlinic configuration. This opens the possibility that test fields which are respectively slowly or rapidly increased from zero will give rise to different configurations, and can in principle provide a route to bistability.

The theory now permits the derivation of an analytic formula for the function $\Phi_{b}(h)$, defined in eq.(4). We obtain

$$
\Phi_{b}=8 \frac{k_{0} n_{e} \nu \alpha h}{\pi^{2}\left(1-h^{2}\right)^{2}}+O\left(\alpha^{2}\right)
$$

that well approximates the small $h$ part of the graph of $\Phi_{b}$ in Figure 3. To capture the maximum of this curve we have to include terms up to $O\left(\alpha^{3}\right)$. This expression can be obtained analytically in terms of $\psi$, rather than $h$ : its expression is not reported here because of its length. However, the closeness of the fit of this solution to the exact numerical values (see Figure 3 ) gives a clear indication that the approximation (6) is valid for a large range of $h$ and $b$.

In summary, the effect of the bias field in magnetic systems is nearly always assumed to be negligible: the bias field is a "useful" field to eliminate unwanted behavior, while not altering significantly the state induced by the much larger test field. We have shown here that this statement must be made with some caution. First of all, a small bias, while not changing the qualitative aspects of the stationary state, may change its quantitative fea- tures significantly. In the particular case of the system studied here the rate of change of the phase lag with the bias field, $\Phi_{b}$, can be quite large and may result in relatively large variations of the test field at fixed phase lag (see Figure 2). If the bias field is sufficiently large, but still small with respect to typical test fields, the changes induced by it may also be qualitative. In a liquid crystal cell, the bias field makes the anticlinic solution accessible experimentally, while the synclinic solution becomes separated from the zero field solution by a potential barrier. These points have to be kept in mind when studying the effect of bias field used to align ferronematic liquid crystals: it can be significantly more than just aligning the ferromagnetic nanoparticles.

We thank Keith Daly for help with software development, Oleksandr Buluy for help with the experiment, Viktor Zadorozhnii, Yuriy Reznikov and Victor Reshetnyak for useful discussions. Financial support for this work was provided by CoI MOD.

[1] P. G. de Gennes and J. Prost, The physics of liquid crystals (Clarendon Press, Oxford, 1993), 2nd ed.

[2] T. J. Sluckin, D. A. Dunmur, and H. Stegemeyer, Crystals that flow: classic papers from the history of liquid crystals (Taylor and Francis, 2004).

[3] O. Buluy, E. Ouskova, Y. Reznikov, A. Glushchenko, J. West, and V. Reshetnyak, Mol. Cryst. Liq. Cryst. 375, 81 (2002).

[4] S. V. Burylov and Y. L. Raikher, Mat. Science Eng. C2, 235 (1995).

[5] V. Zadorozhnii, T. J. Sluckin, V. Y. Reshetnyak, and K. Thomas, SIAM J. Appl. Math. 68, 1688 (2008).

[6] D. V. Makarov, and A. N. Zakhlevnykh, Phys. Rev. E 81, 051710 (2010).

[7] W. Hu, et al., Liq. Cryst. 37, 563 (2010).

[8] L. M. Blinov, Electro-optical and magneto-optical properties of liquid crystals (Wiley, Chichester, 1983).

[9] H. Kawamoto, Proc IEEE 90, 460 (2002).

[10] K. Han, T. Miyashita, and T. Uchida, Jpn. J. Appl. Phys. 32, L277 (1993).

[11] L. N. Trefethen, Spectral Methods in Matlab (SIAM, Philadelphia, 2000).

[12] C. G. Broyden, Math. of Computation 19, 577 (1965), URL http://www. jstor.org/stable/2003941.

[13] H. L. Ong, Phys. Rev. A 31, 3450 (1985).

[14] P. Bos and K. Koehler, Mol. Cryst. Liq. Cryst. 113, 329 (1984).

[15] E. J. Acosta, M. J. Towler, and H. G. Walton, Liq. Cryst. 27, 977 (2000).

[16] P. Glendinning, Stability, Instability and Chaos: An Introduction to the Theory of Nonlinear Differential Equations (Cambridge University Press, Cambridge, UK, 1994). 